\title{
ON THE RESIDUAL FINITENESS OF FREE SEMIGROUPS AND COMPOSITION OF BOOLEAN MATRICES
}

\author{
KI HANG KIM, FRED W. ROUSH AND WOLFGANG SCHÖNFELD
}

By. Boolean matrices are meant matrices over the semiring $\{0,1\}$ under the operations $\sup \{a, b\}$ and $a b$. Such matrices can be regarded as relations on a finite set of individuals. In this way relation algebras or equivalently, certain types of programming languages can be treated by means of operations on matrices. In this note we consider expressions involving only matrix multiplication.

The following proposition yields a new (as far as we know) proof of the residual finiteness of free semigroups. The corollary, when applied to the Boolean algebra $\{0,1\}$ shows that no semigroup identities hold for all square matrix semigroups over this Boolean algebra.

Proposition. Let $l, k$ be positive integers. Let $S$ be the set of l-tuples of integers from 1 to $k$. Let $x_{i}$ be the mapping $S$ to $S$ sending $\left(y_{1}, \ldots, y_{l}\right)$ to $\left(i, y_{1}, \ldots, y_{l-1}\right)$. Then distinct words of length $\leqslant l$ in the transformations $x_{i}$ are distinct transformations.

COROllary. If $R$ is a semiring containing 0 and at least one nonnilpotent element, then no nontrivial identity holds for all semigroups of square matrices over $R$.

Proof. Consider the homomorphism from matrices with only one nonzero element in each row to transformations on a finite set, and use the proposition.

The authors are deeply indebted to the referee for a very helpful suggestion.

Department of Mathematics, Alabama State University, Montgomery, Alabama 36101

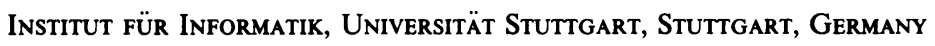

Received by the editors July 7, 1976 and, in revised form, September 21, 1976 and November 8, 1976.

AMS (MOS) subject classifications (1970). Primary 20M05, 02F47; Secondary 15A33, 94A20.

Key words and phrases. Boolean matrix, transformation. 